\title{
Mapping of exciton-exciton annihilation in a molecular dimer via fifth-order femtosecond two-dimensional spectroscopy
}

Cite as: J. Chem. Phys. 150, 104304 (2019); https://doi.org/10.1063/1.5086151

Submitted: 18 December 2018 . Accepted: 14 February 2019. Published Online: 13 March 2019

J. Süß, J. Wehner, J. Dostál, T. Brixner (D), and V. Engel (iD)

\section{ARTICLES YOU MAY BE INTERESTED IN}

Theory of coherent two-dimensional vibrational spectroscopy

The Journal of Chemical Physics 150, 100901 (2019); https://doi.org/10.1063/1.5083966

Where in the world is AIP Publishing? Find out where we are exhibiting next 


\title{
Mapping of exciton-exciton annihilation in a molecular dimer via fifth-order femtosecond two-dimensional spectroscopy
}

\author{
Cite as: J. Chem. Phys. 150, 104304 (2019); doi: 10.1063/1.5086151 \\ Submitted: 18 December 2018 - Accepted: 14 February 2019 • \\ Published Online: 13 March 2019
}

\section{J. Süß, ${ }^{\prime}$ J. Wehner, ${ }^{1}$ J. Dostál, ${ }^{2}$ T. Brixner, ${ }^{2}$ (D) and V. Engel ${ }^{1}$}

\author{
AFFILIATIONS \\ ${ }^{1}$ Institut für Physikalische und Theoretische Chemie, Universität Würzburg, Emil-Fischer-Str. 42, \\ 97074 Würzburg, Germany \\ ${ }^{2}$ Institut für Physikalische und Theoretische Chemie, Universität Würzburg, Am Hubland, \\ 97074 Würzburg, Germany and Center for Nanosystems Chemistry (CNC), \\ Theodor-Boveri-Weg, 97074 Würzburg, Germany
}

\begin{abstract}
We present a theoretical study on exciton-exciton annihilation (EEA) in a molecular dimer. This process is monitored using a fifth-order coherent two-dimensional (2D) spectroscopy as was recently proposed by Dostál et al. [Nat. Commun. 9, 2466 (2018)]. Using an electronic three-level system for each monomer, we analyze the different paths which contribute to the $2 \mathrm{D}$ spectrum. The spectrum is determined by two entangled relaxation processes, namely, the EEA and the direct relaxation of higher lying excited states. It is shown that the change of the spectrum as a function of a pulse delay can be linked directly to the presence of the EEA process.
\end{abstract}

Published under license by AIP Publishing. https://doi.org/10.1063/1.5086151

\section{INTRODUCTION}

Exciton-exciton annihilation (EEA) is a prominent relaxation process which occurs in organic semi-conductors, light-harvesting systems, and molecular aggregates, and it has been discussed vividly in the literature. ${ }^{7-7}$ The microscopic picture behind the EEA process is illustrated, within a basis of localized states, for a dimeric system consisting of two equal monomers $M$, shown in the lefthand and middle-hand panels of Fig. 1. Each monomer possesses three electronic states, namely, its ground $(|g\rangle)$, first $(|e\rangle)$, and second excited states $(|f\rangle)$. The absorption of two photons leads to the population of delocalized dimer eigenstates. For short enough excitation pulses which are spectrally broad, a coherent superposition of eigenstates is excited. The resulting wave packets correspond to states with more or less local character, as discussed in more detail in Sec. II A.

An initially prepared localized state will transform, in the course of time, into other states with local characters. Suppose now that the prepared wave packet corresponds to the localized state $|e e\rangle$ (with configuration $\mathrm{M}^{*} \mathrm{M}^{*}$ ) where both monomers are excited. Thus, electronic excitation energy is present on both sites, i.e., the photo-excitation produces two localized excitons. ${ }^{8-10}$ As time passes, the wave packet moves, and the localized state $|f g\rangle$ (and also $|g f\rangle$, not shown in Fig. 1) with configuration $\mathrm{M}^{* *} \mathrm{M}$ is populated. The state corresponds to a configuration where one monomer is excited to its second excited state $|f\rangle$, whereas the other one is de-excited to its ground state. Upon the usually fast relaxation (with rate $k_{M}$ ), one ends up in the configuration $|e g\rangle$ $\left(\mathrm{M}^{*} \mathrm{M}\right)$ with one monomer in its first excited state and the other one in its ground state. Concerning the energy balance, one finds that one quantum of excitation energy is missing so that an exciton is annihilated.

It is an idealization to assume that the initial wave packet has only the character of the $|e e\rangle$ state. Rather, there are also components corresponding to the localized state $|f g\rangle$ (and also $|g f\rangle$ ). These states undergo relaxation. We refer to this process as direct relaxation (keeping in mind that this involves a photoinduced creation of the $|f g\rangle$ and $|g f\rangle$ states as a first step), and it is illustrated in the right-hand and middle panels of Fig. 1. We address the question if it is possible to disentangle the EEA and the direct relaxation spectroscopically in a dimer. 


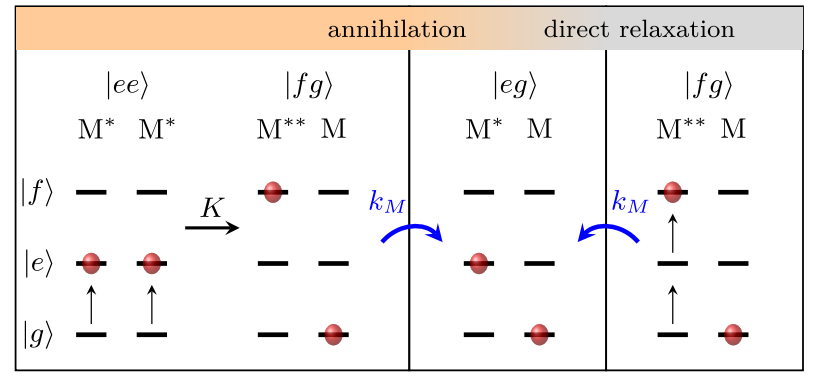

FIG. 1. Relaxation dynamics after light-induced excitations (vertical arrows) in the dimer system. Left and middle panels: Illustration of the EEA process in a localized picture. The picture reflects the structure of the Hamiltonian [Eqs. (2)-(5)] where localized states are coupled. Two singly excited monomers $\left(M^{*} M^{*}\right)$ interact such (via the coupling $K$ ) that one is promoted to its second excited state, whereas the other one is de-excited to the ground state $\left(\mathrm{M}^{* *} \mathrm{M}\right)$. Note that within a wave-packet picture, the initial state $|e e\rangle$ results from the excitation of a coherent superposition of eigenstates which evolves in time into the localized state $|f g\rangle$. In a second step, relaxation takes place where the respective rate is $k_{M}$. Right and middle panels: Direct double excitation on one site is followed by relaxation. Both pathways end up in the same final configuration $\left(M^{*} M\right)$, where one quantum of excitation energy is missing.

Experiments aiming at the characterization of EEA were performed, e.g., on aggregates of perylene bisimide in the liquid phase $^{11-13}$ or conjugated polymers. ${ }^{4,5,14}$ In a theoretical study, Brüggemann and Pullerits have simulated a fifth-order signal in the Fenna-Matthews-Olson complex numerically that carries information on EEA. ${ }^{15}$ Recently, it was shown that it is possible to detect exciton-exciton interactions and to extract diffusion time constants from coherent two-dimensional (2D) spectra. ${ }^{16}$ The EEA is connected to an appearance of spectral peaks during a pulse delay time (population time) and it becomes more effective if the laser fields interacting with a sample are of high intensity. This is due to the fact that, with increasing field strength, the number of prepared excitons becomes larger, and the probability that two excitons meet is enlarged. This field-strength dependency is mapped by different rise times of specific spectral peaks.

In this paper, we explicitly calculate $2 \mathrm{D}$ spectra and analyze how the temporal evolution of $2 \mathrm{D}$ peaks is connected to the annihilation process. In treating a dimer, the dynamics of interest starts when the exciton pair is already formed. It is then clear that the time scale associated with an exciton diffusion process is missing. Whereas Brüggemann and Pullerits determined 2D spectra for two selected times, ${ }^{15}$ our calculations span the entire temporal range where decay processes take place after laser excitation.

In a conventional noncollinear photon-echo $2 \mathrm{D}$ experiment, one records a third-order signal which is triggered by three laser pulses with wave vectors $\vec{k}_{n}$, where the signal in direction $\vec{k}_{s}$ is detected under the phase-matching condition $\vec{k}_{s}=-\vec{k}_{1}+\vec{k}_{2}+\vec{k}_{3}$. The $2 \mathrm{D}$ spectrum then appears in an energy range, both for the excitation and the detection step, that is centered around the photon energy of the pulses being in resonance with the transition from the ground to the first excited state. These 2D techniques have been developed first in nuclear magnetic resonance spectroscopy $^{17,18}$ and later on in the infrared ${ }^{17-25}$ and optical regime. ${ }^{26-33}$ Using coherent optical $2 \mathrm{D}$ spectroscopy, various scientific questions were addressed such as the characterization of intermolecular couplings and conformational states in dimers, ${ }^{34}$ energy transfer ${ }^{35}$ and excitonic structures of photosynthetic systems, ${ }^{36}$ the role of electronic and vibrational coherences, ${ }^{37,38}$ the reaction dynamics in molecular switches, ${ }^{39}$ ultrafast photo-induced charge transfer, ${ }^{40}$ or the dynamics in nanostructures. ${ }^{41,42}$ Here, however, we wish to evaluate not just the single-exciton dynamics following excitation, but to reveal higher-order exciton-exciton interactions (EEI), i.e., following the initial preparation of two excitations within the same system. In general, such higher-order effects overlap with the regular photon-echo-type $2 \mathrm{D}$ spectra and lead to distorted line shapes as well as modified kinetic evolution. Thus, it is difficult (if possible at all) to isolate a signal which reflects the two-exciton interactions. On the other hand, the appropriate fifthorder signal ${ }^{16}$ arises if exciton-exciton interaction is present. Thus, we here address which features of the EEI 2D signal contain which type of information.

To understand the basic idea of the mapping of an EEA process via $2 \mathrm{D}$ spectroscopy, we treat a molecular dimer employing only electronic states, thereby neglecting internal nuclear degrees of freedom. Electronic level models have been used successfully to describe many properties of molecular aggregates. ${ }^{10,43,44}$ This applies to exciton-exciton annihilation processes as well. ${ }^{10,45-49}$ What is not described in detail is the internal conversion and relaxation which proceed via vibrational states. ${ }^{50}$ Also, the appearance of the $2 \mathrm{D}$ spectra will change when including the vibrational motion. ${ }^{51,52}$ In particular, the spectral peaks discussed in Sec. III are no longer resolved.

The analysis of dimers has proved to be crucial if properties of larger aggregates are to be understood. ${ }^{43,44,53}$ A first thorough theoretical study of dimers which included intra-monomer vibrational degrees of freedom was performed by Fulton and Gouterman as early as $1964 .{ }^{54}$ Recently, the so-called dimer approach was introduced which combines high-level quantum chemical calculations on dimeric systems with the quantum dynamical treatments of nuclear degrees of freedom. ${ }^{55}$ In order to deal with multiple excitations correctly, we have to incorporate at least three electronic levels for each monomer and also the effect of an environment on the system. ${ }^{10,45-47}$ The underlying model is described in Sec. II. There, we also present the time-propagation scheme and details on the calculation of the 2D spectra. Numerical results are given in Sec. III, and a summary is presented in Sec. IV.

\section{THEORY AND MODEL}

\section{A. Dimer Hamiltonian}

We regard a homodimer with only electronic degrees of freedom. For each monomer M, three states are taken into account, namely, the electronic ground state $|g\rangle$, a first state $|e\rangle$, and a second excited state $|f\rangle$. The respective energies are $\epsilon_{g}, \epsilon_{e}$, and $\epsilon_{f}$. The dimer Hamiltonian reads

$$
\hat{H}=\hat{H}_{0}+\hat{H}_{1}+\hat{H}_{2}+\hat{H}_{3},
$$

with

$$
\hat{H}_{0}=E_{g g}(|g g\rangle\langle g g|),
$$




$$
\begin{aligned}
\hat{H}_{1}= & E_{e g}(|e g\rangle\langle e g|+| g e\rangle\langle g e|)+J(|e g\rangle\langle g e|+\text { h.c. }), \\
\hat{H}_{2}= & E_{e e}(|e e\rangle\langle e e|)+E_{g f}(|f g\rangle\langle f g|+| g f\rangle\langle g f|) \\
& +K(|f g\rangle\langle e e|+| g f\rangle\langle e e|+\text { h.c. }), \\
\hat{H}_{3}= & E_{e f}(|f e\rangle\langle f e|+| e f\rangle\langle e f|)+L(|f e\rangle\langle e f|+\text { h.c. }),
\end{aligned}
$$

where h.c. is the Hermitian conjugate. The Hamiltonian is represented in the basis of localized electronic monomer states. These localized states differ in the number of excitation quanta in the monomers (see Fig. 1). When the first monomer is in its electronic state $|n\rangle$ and the second in its state $|m\rangle$, the localized dimer state is denoted as $|n m\rangle$. The ground state of energy $E_{g g}=2 \epsilon_{g}$ is $|g g\rangle$. Single excitation leads to the states $|e g\rangle$ and $|g e\rangle$ of equal energy $E_{e g}$ $=\epsilon_{g}+\epsilon_{e}$ which are coupled through the matrix element $J$. The latter results from the electronic interaction of the two locally excited configurations and expressions for the matrix elements are readily available. ${ }^{10}$ Double excitation of a single monomer yields the states $|f g\rangle$ and $|g f\rangle$ (energy $E_{g f}=\epsilon_{g}+\epsilon_{f}$ ), and simultaneous excitation of both monomers results in the state $|e e\rangle$ with energy $E_{e e}=2 \epsilon_{e}$. The latter three configurations interact, where the respective matrix element is $K$ which can be calculated from the Coulomb interaction if the electronic wave functions are available. ${ }^{10}$ We neglect the coupling between $|f g\rangle$ and $|g f\rangle$ which would mediate the transfer of two excitation quanta. Finally, the presence of three excitation quanta leads to states $|f e\rangle$ and $|e f\rangle$ with energies $E_{e f}$ which are also coupled to each other via the interaction of the excited-state configurations (matrix element $L$ ). The Hamiltonian contains contributions from excited states with up to three excitation quanta. Thus, the state $|f f\rangle$ is neglected.

We now switch to the basis of eigenstates of the dimer which are obtained by diagonalization of the dimer Hamiltonian $\hat{H}$ [Eq. (1)] [see Fig. 2 (left)]. Besides the ground state $|G\rangle=|g g\rangle$ (of energy $G=E_{g g}$ ), one finds the one-exciton states which are $( \pm)$ linear combinations of the $|e g\rangle$ and $|g e\rangle$ local states

$$
\left|E_{1}\right\rangle=\frac{1}{\sqrt{2}}(|e g\rangle-|g e\rangle),\left|E_{2}\right\rangle=\frac{1}{\sqrt{2}}(|e g\rangle+|g e\rangle),
$$

and the eigenenergies are

$$
E_{1}=E_{\text {eg }}-J, \quad E_{2}=E_{\text {eg }}+J .
$$

The three states $\left|D_{n}\right\rangle$ of the second excited-state manifold with energies $D_{n}$ are linear combinations of the $|f g\rangle$, $|e e\rangle$ and $|g f\rangle$ states $(n=1-3)$,

$$
\left|D_{n}\right\rangle=c_{n 1}|f g\rangle+c_{n 2}|e e\rangle+c_{n 3}|g f\rangle .
$$

The coefficients are functions of the monomer eigenenergies and the coupling $K$, and they are not given explicitly here. The respective eigenenergies are

$$
\begin{aligned}
& D_{1}=\frac{1}{2}\left(E_{e e}+E_{g f}-\sqrt{\left(E_{e e}-E_{g f}\right)^{2}+8 K^{2}}\right), \\
& D_{2}=E_{g f}, \\
& D_{3}=\frac{1}{2}\left(E_{e e}+E_{g f}+\sqrt{\left(E_{e e}-E_{g f}\right)^{2}+8 K^{2}}\right) .
\end{aligned}
$$

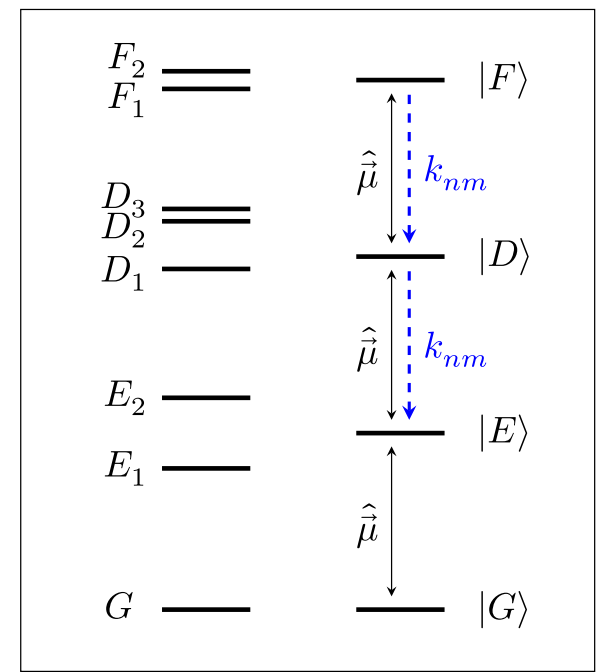

FIG. 2. Level scheme of the dimer model. The left-hand side shows the order of the eigenenergies of the dimer Hamiltonian. The right-hand side illustrates the excitation scheme. The laser excitation (indicated by the dipole operator $\hat{\vec{\mu}}$ ) couples the ground $(|G\rangle)$ state with the manifold of first excited states $\left(|E\rangle\right.$, energies $\left.E_{1}, E_{2}\right)$, the latter with the manifold of second excited states $\left(|D\rangle\right.$, energies $\left.D_{1}, D_{2}, D_{3}\right)$, and also the second excited states with the two third excited states $(|F\rangle$, energies $\left.F_{1}, F_{2}\right)$. The $|F\rangle$ and $|D\rangle$ states decay non-radiatively with the state-to-state rates $k_{n m}$.

Finally, the third excited states are $( \pm)$ combinations of $|e f\rangle$ and $|f e\rangle$,

$$
\left|F_{1}\right\rangle=\frac{1}{\sqrt{2}}(|e f\rangle-|f e\rangle), \quad\left|F_{2}\right\rangle=\frac{1}{\sqrt{2}}(|e f\rangle+|f e\rangle)
$$

with energies

$$
F_{1}=E_{e f}-L, \quad F_{2}=E_{e f}+L .
$$

The level structure of the dimer is illustrated in the left-hand part of Fig. 2 and involves eight states of different energies.

Let us comment on the relation between the localized states and the eigenstates in the connection with a femtosecond pulse experiment as described in this paper. Because the pulses are energetically broad, linear combinations of the eigenstates are prepared by the field-matter interaction. These wave packets are of more or less localized character. Regard, as a simple example, an excitation of a wave packet $|\psi(t)\rangle$ consisting of the $\left|E_{1}\right\rangle$ and $\left|E_{2}\right\rangle$ states

$$
|\psi(t)\rangle=a_{1} e^{-\frac{i}{\hbar} E_{1} t}\left|E_{1}\right\rangle+a_{2} e^{-\frac{i}{\hbar} E_{2} t}\left|E_{2}\right\rangle,
$$

with coefficients $a_{1}$ and $a_{2}$. Switching to the local basis, we have

$$
\begin{aligned}
|\psi(t)\rangle= & \left(\frac{a_{1}}{\sqrt{2}} e^{-\frac{i}{\hbar} E_{1} t}+\frac{a_{2}}{\sqrt{2}} e^{-\frac{i}{\hbar} E_{2} t}\right)|e g\rangle \\
& +\left(-\frac{a_{1}}{\sqrt{2}} e^{-\frac{i}{\hbar} E_{1} t}+\frac{a_{2}}{\sqrt{2}} e^{-\frac{i}{\hbar} E_{2} t}\right)|g e\rangle .
\end{aligned}
$$


The local character of the component $|e g\rangle$ being present in the wave packet is characterized by calculating the population of this state by projection

$$
P_{e g}(t)=|\langle e g \mid \psi(t)\rangle|^{2}=\frac{\left|a_{1}\right|^{2}+\left|a_{2}\right|^{2}}{2}+\left|a_{1}\right|\left|a_{2}\right| \cos \left[\left(E_{2}-E_{1}\right) t / \hbar+\beta\right],
$$

where $\beta$ is the relative phase between the coefficients $a_{1}$ and $a_{2}$. Thus, the population in the local configuration $|e g\rangle$ oscillates as a function of time where the oscillation period is given by the energy spacing of the two states being present in the wave packet. In this example, the population in the second component $|g e\rangle$ is just $P_{g e}(t)=\left|a_{1}\right|^{2}$ $+\left|a_{2}\right|^{2}-P_{e g}(t)$, so there is a transfer of population between the two local configurations. Depending on the relative magnitude of the coefficients, the population dynamics shows more or less temporal variations superimposed on a background.

A similar consideration concerning the population of localized components can be applied to the linear combination of the states $\left|D_{n}\right\rangle$. This brings us to the following interpretation of Fig. 1: A femtosecond excitation involving the absorption of two photons prepares an initial wave packet consisting of the coherent superposition of the three states $\left|D_{n}\right\rangle$. At some time, the localized state $|e e\rangle$ $\left(\mathrm{M}^{*} \mathrm{M}^{*}\right)$ is populated. As time passes, the local character of the wave packet changes and the population of the component $|f g\rangle\left(\mathrm{M}^{* *} \mathrm{M}\right)$ increases; afterwards, annihilation can occur.

We restrict our calculations to configurations where all transition dipole moments of the monomers are aligned parallel to each other and are orthogonal to the vector between the centers of mass of the two monomers. ${ }^{43,44}$ The couplings are then taken as positive (being consistent with the Förster coupling ${ }^{10}$ ). According to the positive value of the coupling $J$, this corresponds to an $\mathrm{H}$-aggregate. We note that, for dimers and degenerate levels $E_{f g}=E_{e e}$, the annihilation rate is similar for $\mathrm{H}$ - and J-aggregates. ${ }^{56}$

The parameters entering into the dimer Hamiltonian are summarized in Table I. These are the monomer energies and the couplings $J, K$, and $L$. Also given is a value for the relaxation rate $k_{M}$ for the $|e\rangle \leftarrow|f\rangle$ monomer transition. The chosen value yields a lifetime of $67 \mathrm{fs}$ for the second excited state. In the context of

TABLE I. Parameters employed in the numerical calculations. The monomer energies are denoted as $\epsilon_{g}, \epsilon_{e}, \epsilon_{f}$ and $J, K, L$ are coupling constants. The relaxation rate from the second to the first excited monomer state is $k_{M}$. Relaxation from the first excited monomer state to the ground state is not included so that the respective rate $\tilde{k}_{M}$ is equal to zero.

\begin{tabular}{lccc}
\hline \hline & $\epsilon_{g}$ & $\epsilon_{e}$ & $\epsilon_{f}$ \\
Energy $(\mathrm{eV})$ & 0.0 & 1.0 & 2.2 \\
\hline \multirow{2}{*}{ Coupling $(\mathrm{eV})$} & $J$ & $K$ & $L$ \\
\hline & 0.2 & 0.1 & 0.05 \\
Rate $\left(\mathrm{fs}^{-1}\right)$ & $k_{M}$ & $\tilde{k}_{M}$ & \\
\hline \hline
\end{tabular}

exciton-exciton annihilation, similar values have been reported; see, e.g., Refs. 46, 48, and 49. The finite lifetime introduces a line broadening which is in the order of $10 \mathrm{meV}$. We note that the simultaneously occurring processes of internal conversion and relaxation are determined by transitions which involve the energetically close lying vibrational states in the second and first electronically excited states. Within our electronic level model, an energy of about $1 \mathrm{eV}$ is transferred to the surrounding. The bath does not absorb this energy in one quantum, rather the energy transfer takes place successively in dissipating smaller quanta of vibrational energies. Relaxation from the first excited monomer states to their ground states $\left(\tilde{k}_{M}\right)$ is not included because it typically occurs on time scales longer than those we consider in the present study of short-time dynamics.

The energy of the $|f g\rangle$ state is roughly twice the energy of the $|e g\rangle$ state. This is motivated by the fact that we are interested in spectra which exhibit intensity in the region of twice the excitation energy of the first electronic transition. It is a condition for exciton-exciton annihilation that a single-exciton (doubly excited) state is available that has about twice the energy of a oneexciton (singly excited) state because only then the initial step of the EEA process happens under energy conservation. Usually, due to a high density of states in larger molecules, a resonant transition is possible at this energy. Note that this is also possible in atomic systems. ${ }^{57}$

The coupling constants $J, K$, and $L$ are chosen such that the $2 \mathrm{D}$ spectra exhibit several well resolved peaks. The value of $J$ determines the splitting of the one-exciton states $E_{1}$ and $E_{2}$, but the eigenstates and thus the rates $k_{n m}$ for transitions into these states do not depend on $J$ (see Sec. II B). Thus, the strength of this coupling has no influence on the dynamics of the annihilation process. The same holds for the coupling $L$ and transitions involving the eigenstates $\left|F_{1}\right\rangle,\left|F_{2}\right\rangle$. The strength of the coupling $K$, however, is important because it directly determines the efficiency of the EEA process.

\section{B. Propagation}

For the time propagation of the dimer system coupled to an environment, we solve a stochastic Schrödinger equation. ${ }^{58,59}$ From the ensemble of stochastic wave functions obtained within this approach, the reduced density matrix of a system can be constructed. It is then possible to calculate the expectation value of any system operator. The adaption of the quantum-jump approach to calculate $2 \mathrm{D}$ spectra is described in detail in Ref. 60, and here we briefly summarize the working equations needed. A simplified version of the propagation scheme is applied which neglects dephasing processes.

As detailed further, we allow relaxation from the second excited states $\left|D_{n}\right\rangle$ to the singly excited states $\left|E_{m}\right\rangle$ and also from the third excited states $\left|F_{n}\right\rangle$ to the second excited states $\left|D_{n}\right\rangle$; see Fig. 2 (right). The model does not incorporate intraband relaxation. Concerning the annihilation dynamics, such dissipative processes taking place between the $|E\rangle$ states do not have a pronounced effect because when an $|E\rangle$ state is reached, no further annihilation takes place. On the other hand, intraband relaxation within the $|D\rangle$ manifold might be of importance because it eliminates possibly existing coherences on the time scale of this process. If the lowest eigenstate $\left|D_{1}\right\rangle$ 
is reached, annihilation is blocked because it is no longer possible to exchange population between the $|e e\rangle,|f g\rangle$ and $|g f\rangle$ local configurations.

The stochastic propagation scheme described here is applied to the manifolds of excited states. The ground state $|G\rangle$ which cannot be reached by relaxation processes develops in time as a stationary state, i.e., with the phase factor $\exp \left(-\frac{i}{\hbar} G t\right)$. Denoting the eigenstates as $|n\rangle$ and the respective energies as $E_{n}$ leads to the expansion of the excited-state wave packet as

$$
|\psi(t)\rangle=\sum_{n} c_{n}(t)|n\rangle,
$$

with expansion coefficients $c_{n}(t)$ and where $n=\left\{E_{1}, E_{2}, D_{1}, D_{2}, D_{3}\right.$, $\left.F_{1}, F_{2}\right\}$. We note that because the calculation of the $2 \mathrm{D}$ spectra is performed within perturbation theory, the norm of $|\psi(t)\rangle$ is not equal to one. In order to apply the stochastic propagation scheme, it is necessary to normalize it. This is done at each time step, and the original norm is restored after the propagation step.

For the short-time propagator, which advances the state for a time step $d t$, two possible realizations exist. A jump from an initial state $|n\rangle$ to a final state $|m\rangle$ takes place with the probability $p_{j}(t)$, where $j$ indicates "jump," leading to

$$
|\psi(t+d t)\rangle=\frac{c_{n}(t)}{\left|c_{n}(t)\right|}|m\rangle .
$$

The jumps take place between the eigenstates and are not to be confused with any kind of hopping process between the different monomer units. In our calculation, only downward jumps are included. The second possibility is a coherent propagation where the respective probability is $p_{c}(t)$, where $c$ indicates "coherent," and the state vector is advanced as

$$
|\psi(t+d t)\rangle=\frac{1}{\sqrt{c(t)}} \sum_{n} c_{n}(t) e^{-\frac{i}{\hbar} E_{n} d t-\Gamma_{n} d t / 2}|n\rangle,
$$

with

$$
c(t)=1-d t \sum_{n} \Gamma_{n}\left|c_{n}(t)\right|^{2}+\mathcal{O}\left(d t^{2}\right)
$$

and $\Gamma_{n}$ are escape rates (defined below). The coherent propagation step is norm conserving to first order in $d t$.

The numbers $p_{j}(t)$ and $p_{c}(t)$ are fixed as follows. The jump rate between the states $|n\rangle$ and $|m\rangle$ is denoted as $k_{n m}$, and the state-tostate jump probability is

$$
p_{n m}(t)=k_{n m}\left|c_{n}(t)\right|^{2} d t .
$$

This yields the total jump probability

$$
p_{j}(t)=\sum_{n} \sum_{m \neq n} p_{n m}(t),
$$

and the probability for the coherent propagation is

$$
p_{c}(t)=1-p_{j}(t)=c(t) .
$$

Furthermore, the escape rates are defined as

$$
\Gamma_{n}=\sum_{m \neq n} k_{n m} .
$$

As mentioned in the Introduction, the EEA involves a radiationless transition between the monomer states $|f\rangle$ and $|e\rangle$. We take the corresponding rate $k_{M}$ as a parameter. Relaxation processes between the first excited-state manifold and the ground state are neglected because, according to Kasha's rule, ${ }^{61}$ they usually proceed on a much longer time scale than regarded here. Note also that we treat only transitions between the different manifolds of excited electronic states. This assumes that intraband relaxation takes place on a much longer time scale.

To calculate the transition rates $k_{n m}$, the mixing of the states $|f g\rangle,|g f\rangle,|f e\rangle$, and $|e f\rangle$ into the initial $(|n\rangle)$ and final state $(|m\rangle)$ has to be taken into account, and we define

$$
\begin{aligned}
k_{n m}= & \left(|\langle n \mid f g\rangle|^{2}|\langle e g \mid m\rangle|^{2}+|\langle n \mid g f\rangle|^{2}|\langle g e \mid m\rangle|^{2}\right. \\
& \left.+\left[|\langle n \mid f e\rangle|^{2}+|\langle n \mid e f\rangle|^{2}\right]|\langle e e \mid m\rangle|^{2}\right) k_{M} .
\end{aligned}
$$

For the propagation, we calculate the probabilities $p_{n m}(t)$ and $p_{c}(t)$ at each time $t$ and arrange these numbers in the unit interval $[0,1]$. Within a Monte Carlo sampling, a random number drawn from a uniformly distributed pool determines the next step in the propagation scheme. The time step has to be chosen small enough to ensure that the jump probabilities are much smaller than one. In our numerical calculation, we used $d t=0.3 \mathrm{fs}$ and a number of $N_{r}=1500$ runs to yield convergent results. The described stochastic propagation applies to the excited-state wave packet. The ground state is treated differently because there, only the coherent time evolution takes place. The quantum-jump scheme enables us to evolve a wave packet for one time step. To start the propagation, initial conditions have to be specified. They are connected to the laser excitation process and will be discussed in Sec. II C.

Repeating the calculation $N_{r}$ times yields the reduced density operator for the dimer

$$
\hat{\rho}(t)=\frac{1}{N_{r}} \sum_{l=1}^{N_{r}}\left|\psi_{l}(t)\right\rangle\left\langle\psi_{l}(t)\right|,
$$

where $\left|\psi_{l}(t)\right\rangle$ now denotes the total state of the system and $l$ labels the single runs. It can be shown ${ }^{59}$ that the matrix elements of $\hat{\rho}(t)$ [Eq. (26)] fulfill the Redfield equations within the secular approximation. ${ }^{62}$ The expectation values for any system operator $\hat{O}$ then is

$$
\langle\hat{O}\rangle(t)=\frac{1}{N_{r}} \sum_{l=1}^{N_{r}}\left\langle\psi_{l}(t)|\hat{O}| \psi_{l}(t)\right\rangle .
$$

Thus, the outlined stochastic approach can be used to calculate $2 \mathrm{D}$ signals ${ }^{60,63}$ as is usually done using the density-matrix description. ${ }^{64-66}$

\section{2D signals}

The monomer transition dipole moments between the ground and first excited states of monomer $m$ are denoted as $\vec{\mu}_{e g, m}$, and those between the second and third state as $\vec{\mu}_{f e, m}$. The dimer transitiondipole operator in the localized basis is 


$$
\begin{aligned}
\hat{\vec{\mu}}^{l}= & \left(|e g\rangle \vec{\mu}_{e g, 1}\langle g g|+| g e\rangle \vec{\mu}_{e g, 2}\langle g g|+| e e\rangle \vec{\mu}_{e g, 1}\langle g e|+| e e\rangle \vec{\mu}_{e g, 2}\langle e g|\right. \\
& +|e f\rangle \vec{\mu}_{e g, 1}\langle g f|+| f e\rangle \vec{\mu}_{e g, 2}\langle f g|+| f g\rangle \vec{\mu}_{f e, 1}\langle e g| \\
& \left.+|g f\rangle \vec{\mu}_{f e, 2}\langle g e|+| f e\rangle \vec{\mu}_{f e, 1}\langle e e|+| e f\rangle \vec{\mu}_{f e, 2}\langle e e|\right)+ \text { h.c. }
\end{aligned}
$$

The total electric field is the sum of three pulses

$$
\vec{E}(t)=\sum_{l=1}^{3}\left(\vec{E}_{l}\left(+\vec{k}_{l}, t\right)+\vec{E}_{l}\left(-\vec{k}_{l}, t\right)\right),
$$

with the components

$$
\vec{E}_{l}\left( \pm \vec{k}_{l}, t\right)=\frac{\vec{\epsilon}}{2} g_{l}\left(t-T_{l}\right) e^{\mp i \omega_{l}\left(t-T_{l}\right)} e^{ \pm \overrightarrow{k_{l}} \vec{r}_{l} \vec{r}} .
$$

Here, the polarization vectors $\vec{\epsilon}$ are taken as equal for all pulses. The center frequencies are $\omega_{l}$, and the pulse envelopes $g_{l}\left(t-T_{l}\right)$ are centered at times $T_{l}$. In the numerical examples, we employ the impulsive limit for the pulses, i.e., we use $g_{l}\left(t-T_{l}\right)=\mathcal{A}_{l} \delta\left(t-T_{l}\right)$, with the field strengths $\mathcal{A}_{l}$. If not stated differently, the moleculefield coupling takes a value of $\left|\mu_{e g, m} \mathcal{A}_{l}\right|=\left|\mu_{f e, m} \mathcal{A}_{l}\right|=0.5 \mathrm{eV}$. Because we use perturbation theory and calculate normalized quantities, the actual value for this coupling is not of importance. Calculations with finite pulses of $5 \mathrm{fs}$ (full width at half maximum) yield results which do not differ essentially from the ones presented in Sec. III. Within the dipole approximation, the dependence of the field on the coordinate vector $\vec{r}$ is neglected. We include the respective phase factor in Eq. (30) to identify the radiative transitions contributing to the phase-matched signal, see below. Using the rotating-wave approximation, ${ }^{67}$ which is employed in the numerical calculations, the fields $\vec{E}_{l}\left(+\vec{k}_{l}, t\right)$ and $\vec{E}_{l}\left(-\vec{k}_{l}, t\right)$ belong to the processes of absorption and stimulated emission, respectively.

Given the dipole operator and the fields, the time-dependent interaction term is

$$
\hat{W}^{l}(t)=-\hat{\vec{\mu}}^{l} \cdot \vec{E}(t) .
$$

Because we work in the basis of eigenstates, the transition-dipole operator $\hat{\vec{\mu}}^{l}$ is transformed into the same basis yielding the operator $\hat{\mu}$ which couples the eigenstates, where, initially, we start in the ground state $|G\rangle$. The perturbation induces a polarization in the system which reads

$$
\vec{P}(t)=\langle\psi(t)|\hat{\vec{\mu}}| \psi(t)\rangle,
$$

where the state vector $|\psi(t)\rangle$ describes the time evolution of the system. We are interested in the fifth-order contribution to the polarization projected on the detection vector $\vec{\epsilon}_{d}$ which is taken to be parallel to the laser polarization $\vec{\epsilon}$

$$
P^{(5)}(t)=\sum_{n=0}^{5}\left\langle\psi^{(n)}(t)\left|\vec{\epsilon}_{d} \cdot \hat{\vec{\mu}}\right| \psi^{(5-n)}(t)\right\rangle .
$$

The states $\left|\psi^{(n)}(t)\right\rangle$ which enter into the latter expression are determined in $n$th order perturbation theory. An additional restriction to the number of contributions is the phase-matching condition $\vec{k}_{s}=-2 \vec{k}_{1}+2 \vec{k}_{2}+\vec{k}_{3}$ which we impose. In what follows, only paths with the time order $T_{1}<T_{2}<T_{3}$ are considered. The coherence time is defined as $\tau=T_{2}-T_{1}$ and the population time as $T=T_{3}-T_{2}$.
An additional time variable used further below is the detection time $t^{\prime}$ which has its origin at the center of the third pulse.

Within the stochastic approach, a single run leads to the state vector

$$
\left|\psi_{l}(t)\right\rangle=\sum_{n=0}^{5}\left|\psi_{l}^{(n)}(t)\right\rangle,
$$

and the polarization is calculated by an average over $N_{r}$ runs as

$$
P_{s}^{(5)}\left(t^{\prime}, T, \tau\right)=\frac{1}{N_{r}} \sum_{l=1}^{N_{r}} \sum_{n=0}^{5}\left\langle\psi_{l}^{(n)}\left(t^{\prime}, T, \tau\right)\left|\vec{\epsilon}_{d} \cdot \hat{\vec{\mu}}\right| \psi_{l}^{(5-n)}\left(t^{\prime}, T, \tau\right)\right\rangle,
$$

where the dependence of the polarization on the three time variables is explicitly noted. Details of the numerical propagation scheme for the perturbative states $\left|\psi_{l}^{(n)}(t)\right\rangle$ are given in Ref. 60 .

From the polarization, we obtain the $2 \mathrm{D}$ spectrum via $2 \mathrm{D}$ Fourier transformation as ${ }^{68}$

$$
S\left(E_{t^{\prime}}, T, E_{\tau}\right)=i \int d t^{\prime} \int d \tau e^{\frac{i}{\hbar} E_{t^{\prime}} t^{\prime}} e^{-\frac{i}{\hbar} E_{\tau} \tau} \tilde{P}_{s}^{(5)}\left(t^{\prime}, T, \tau\right),
$$

with the energy axes $E_{\tau}$ for the excitation and $E_{t^{\prime}}$ for the detection step. Here, the polarization $\tilde{P}_{s}^{(5)}\left(t^{\prime}, T, \tau\right)$ contains only the contributions which satisfy the phase-matching condition. The spectra are averaged over molecular orientations relative to the field vector in discretizing the orientational angle. In the present case, where we assume an all-parallel transition dipole geometry this yields an overall factor. For different geometries, however, one has to perform the average explicitly because different contributions to the signal are weighted differently. ${ }^{52}$

The paths contributing to the signal are conventionally illustrated using double-sided Feynman diagrams. ${ }^{64,66}$ In the present case, one finds seven diagrams ${ }^{15,16}$ which are collected in Fig. 3 . There, transitions are indicated between the dimer states $|G\rangle,|E\rangle$, $|D\rangle$, and $|F\rangle$. The respective matrix elements which enter into the polarization can be identified as (where we skip the time arguments and the index, labeling the different stochastic runs)

$$
\begin{array}{cl}
\mathrm{I}: & \left\langle\psi_{G}^{(4)}\left(-2 \vec{k}_{1}+2 \vec{k}_{2}\right)\left|\vec{\epsilon}_{d} \cdot \hat{\vec{\mu}}\right| \psi_{E}^{(1)}\left(\vec{k}_{3}\right)\right\rangle, \\
\mathrm{II}: & \left\langle\psi_{E}^{(3)}\left(-2 \vec{k}_{1}+\vec{k}_{2}\right)\left|\vec{\epsilon}_{d} \cdot \hat{\vec{\mu}}\right| \psi_{D}^{(2)}\left(\vec{k}_{2}+\vec{k}_{3}\right)\right\rangle, \\
\mathrm{III}: & \left\langle\psi_{G}^{(4)}\left(-2 \vec{k}_{1}+\vec{k}_{2}+\vec{k}_{3}\right)\left|\vec{\epsilon}_{d} \cdot \hat{\vec{\mu}}\right| \psi_{E}^{(1)}\left(\vec{k}_{2}\right)\right\rangle, \\
\mathrm{IV}: & \left\langle\psi_{E}^{(3)}\left(-2 \vec{k}_{1}+\vec{k}_{3}\right)\left|\vec{\epsilon}_{d} \cdot \hat{\vec{\mu}}\right| \psi_{D}^{(2)}\left(2 \vec{k}_{2}\right)\right\rangle, \\
\mathrm{V}: & \left\langle\psi_{D}^{(2)}\left(-2 \vec{k}_{1}\right)\left|\vec{\epsilon}_{d} \cdot \hat{\vec{\mu}}\right| \psi_{F}^{(3)}\left(2 \vec{k}_{2}+\vec{k}_{3}\right)\right\rangle, \\
\mathrm{VI}: & \left\langle\psi_{G}^{(3)}\left(-2 \vec{k}_{1}+\vec{k}_{3}\right)\left|\vec{\epsilon}_{d} \cdot \hat{\vec{\mu}}\right| \psi_{E}^{(2)}\left(2 \vec{k}_{2}\right)\right\rangle, \\
\mathrm{VII}: & \left\langle\psi_{E}^{(2)}\left(-2 \vec{k}_{1}\right)\left|\vec{\epsilon}_{d} \cdot \hat{\vec{\mu}}\right| \psi_{D}^{(3)}\left(2 \vec{k}_{2}+\vec{k}_{3}\right)\right\rangle .
\end{array}
$$

Here, the ket state $\left|\psi_{X}^{\left(n+n^{\prime}\right)}\left(n \vec{k}_{2}+n^{\prime} \vec{k}_{3}\right)\right\rangle$ is created by absorption of $n$ photons from pulse $2\left(\vec{k}_{2}\right)$ and $n^{\prime}$ photons from pulse $3\left(\vec{k}_{3}\right)$. The bra state $\left\langle\psi_{X}^{\left(2+n+n^{\prime}\right)}\left(-2 \vec{k}_{1}+n \vec{k}_{2}+n^{\prime} \vec{k}_{3}\right)\right|$ results from 

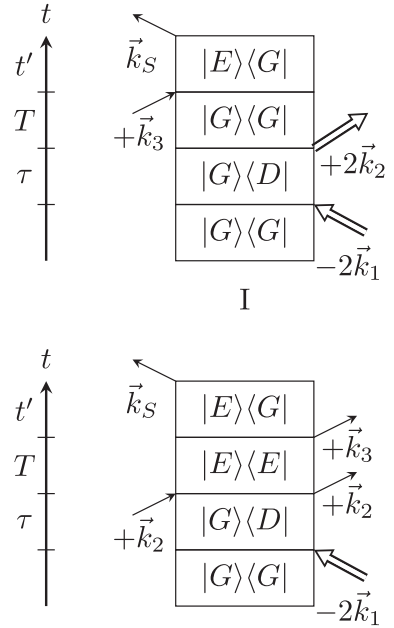

III
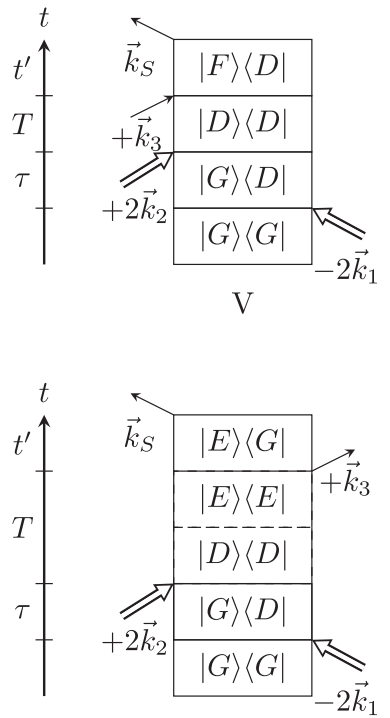

VI

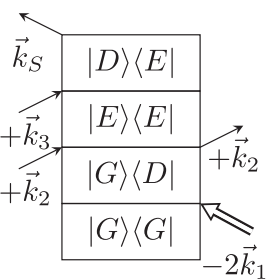

II

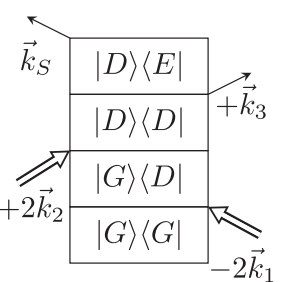

IV

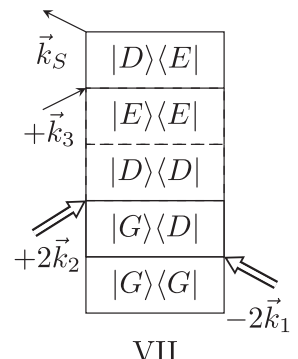

FIG. 3. Double-sided Feynman diagrams illustrating the fifth-order processes leading to the 2D spectra under the phase-matching condition $\vec{k}_{s}=-2 \vec{k}_{1}+2 \vec{k}_{2}+\vec{k}_{3}$. They belong to the matrix elements I-VII as specified in the text. The time ordering is from bottom to top. Absorption and emission from the pulses $\vec{k}_{n}$ are indicated by arrows pointing towards and away from the box, respectively. Transitions take place between the dimer states $|G\rangle,|E\rangle,|D\rangle$ and $|F\rangle$. Diagram $V$ is the only one which involves the third excited states $|F\rangle$. Note that the paths $\mathrm{VI}$ and $\mathrm{VII}$ become only possible if relaxation from $|D\rangle$ is taken into account (indicated by the dashed lines).

two-photon excitation from the first pulse $\left(\vec{k}_{1}\right)$ and $n / n^{\prime}$-photon stimulated emission initiated by pulse $2 / 3$, respectively. The subscript $X$ takes the values $|G\rangle,|E\rangle,|D\rangle$, and $|F\rangle$. Of course, at later times, the states with $X=D, F$ can further relax into the $|E\rangle$ states. If no relaxation from the $|D\rangle$ states takes place, the paths I-V are the only ones possible. However, the two additional paths (VI, VII) occur if the relaxation becomes effective.

\section{RESULTS}

To get an insight into the relaxation dynamics of the dimer system, we first regard the energy expectation values

$$
E_{t o t}(t)=\frac{1}{N_{r}} \sum_{l=1}^{N_{r}} \frac{\left\langle\psi_{l}(t)\left|\hat{H}_{1}+\hat{H}_{2}\right| \psi_{l}(t)\right\rangle}{\left\langle\psi_{l}(t) \mid \psi_{l}(t)\right\rangle}
$$

with Hamiltonians defined in Eqs. (3) and (4), where the ground state and the third excited states do not enter. Starting from the ground state $|G\rangle$, the energy is calculated for times $t$ after the interaction with the first two pulses both acting impulsively at time $t=0$. The populated states which contribute to $E_{\text {tot }}(t)$ can be identified from Fig. 3 in setting the interactions with the third pulse $\left(\vec{k}_{3}\right)$ equal to zero. In Fig. 4, we compare calculations with zero and non-zero values of the coupling $K$, leaving all other parameters the same. For $K$ equal to zero, the eigenstates of the Hamiltonian $\hat{H}_{2}$ [Eq. (4)] are the local states $|e e\rangle,|f g\rangle$, and $|g f\rangle$. Then, because we do not include relaxation between these states, the local state $|e e\rangle$ cannot evolve into the $|f g\rangle$ or $|g f\rangle$ state so that the annihilation channel is closed. Our approach here is that of a reference system which deviates as little as possible from the one to be understood. Formally, this means that we divide the Hamiltonian in that for the reference system (say, $\hat{H}_{r}$ ) and the rest $\left(\hat{H}_{p}\right)$. In our case, $\hat{H}_{p}$ is that part of $\hat{H}_{2}$ [Eq. (4)] which contains the coupling $K$. The properties of the reference system are similar to the total one. The difference is the level splitting in the $|D\rangle$ manifold and the respective eigenfunctions. The consequence of this degeneracy is that the annihilation does not take place. This allows us to get some insight about a system which is excited similar to the total one but decays exclusively via the direct relaxation pathway (see Fig. 1).

For the $K=0$ case, we find an exponential decay of the energy $E_{\text {tot }}(t)$. The decay can be assigned to the relaxation of the

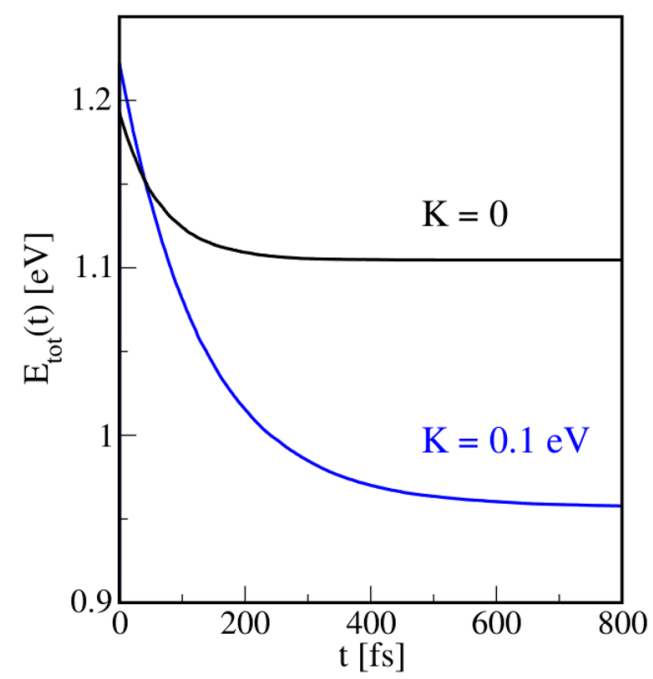

FIG. 4. Energy expectation values of the excited-state manifold for zero and finite coupling $K$, as indicated. The excited-state wave packet at time $t=0$ is prepared by impulsive excitation with the first and second pulse. 
populated $|f g\rangle$ and $|g f\rangle$ components to $|e g\rangle$ and $|g e\rangle$, respectively. Because there is no relaxation channel for the $|e e\rangle$ component, which is an eigenstate, the energy expectation value converges to a value mainly determined by the population in this state. Changing the coupling to a value of $K=0.1 \mathrm{eV}$ activates the EEA process. Due to the finite value of $K$, the initial energy is slightly larger than in the $K=0$ case. One reason for this is that the energies of the $|D\rangle$ states are different from the $K=0$ case [see Eqs. (9)-(11)]. Furthermore, the eigenfunctions and also their amplitudes after excitation differ. This results in a shift of the expectation value which, in the present case, is larger. The decay no longer proceeds monoexponentially. The first step of EEA now prompts the indirect relaxation of the $|e e\rangle$ components. This leads to an overall lower energy expectation value asymptotically. The long-time limit of the energy is somewhere close to the eigenenergies of the $\left|E_{1}\right\rangle$ and $\left|E_{2}\right\rangle$ states which, within the chosen model, are not allowed to decay further.

We next turn to the 2D spectroscopy. In Fig. 5, spectra are displayed in the range around $\left(E_{\tau}, E_{t^{\prime}}\right)=\left(2 \omega_{l} \hbar, \omega_{l} \hbar\right)$, where $\omega_{l}$ is the

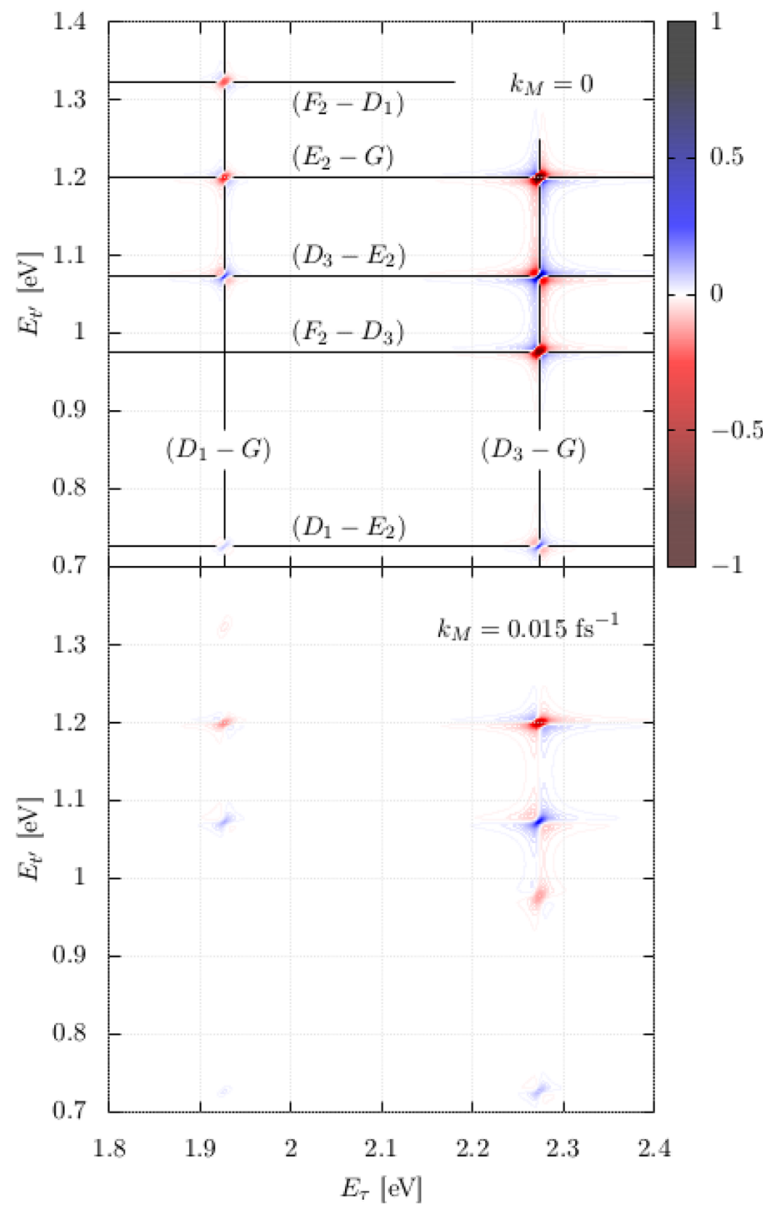

FIG. 5. Real part of 2D spectra for the isolated model (upper panel) and including system-bath interactions (lower panel). The spectra are normalized to the largest peak obtained in the isolated dimer. laser center frequency. The $2 \mathrm{D}$ spectra are calculated using a time interval from zero to $823 \mathrm{fs}$ in both time variables $\left(t^{\prime}, \tau\right)$. The population time is set to $T=0$. The upper panel contains a spectrum obtained for the isolated dimer, where the coupling to the bath is switched off $\left(k_{M}=0\right)$. To avoid noise introduced by Fourier transforming a non-periodic function, the time data is multiplied with Gaussian window functions. In this way, an artificial peak broadening is introduced which is the same for each peak. The spectrum is normalized such that its most intense peak assumes a value of one. In general, peak positions coincide with differences between eigenenergies of the eigenstates excited by the ultrashort pulses. Along the $E_{\tau}$ axis, three peak positions are expected. However for the chosen parallel dipole geometry, the $\left|D_{2}\right\rangle$ state is dark and peaks are present only at energies $E_{\tau}=D_{1}-G$ and $E_{\tau}=D_{3}-G$. On the $E_{t^{\prime}}$ axis, more peak positions occur, as is indicated in the figure.

Including relaxation leads to pronounced spectral changes as can be inferred from the spectrum shown in the lower panel of Fig. 5. The spectrum is normalized to the one shown in the upper panel of the figure; i.e., we use the same normalization constant as for $k_{M}=0$. It is seen that the system-bath coupling results in an intensity loss of the spectrum. Because the eigenstates $\left|F_{n}\right\rangle$ and $\left|D_{n}\right\rangle$ are de-populated due to relaxation, all peaks decay relative to the relaxation-free situation but, in general, with different rates. The peaks involving the energy difference of two decaying states in the $E_{t^{\prime}}$ direction lose more intensity. These peaks are no longer seen in the $2 \mathrm{D}$ spectrum.

The analytical expressions for the time-dependent polarization in the relaxation-free case show that it contains terms which oscillate with phase factors $e^{ \pm i\left(D_{3}-D_{1}\right) T / \hbar}$. This means that the spectra exhibit coherences as a function of the population time. Such oscillations were found in 2D spectra detected within the third-order photon-echo arrangement. ${ }^{38,69-73}$ To document this dependence, we calculate spectra using the temporal interval of $[0,115]$ fs for the coherence and detection time, and we vary the population time from zero to $350 \mathrm{fs}$. For each time $T$, the $2 \mathrm{D}$ spectrum is integrated in the intervals $[1.8,2.4] \mathrm{eV}$ and $[0.6,1.4] \mathrm{eV}$ along $E_{\tau}$ and $E_{t^{\prime}}$, respectively. The resulting energy-averaged quantity then is

$$
\bar{\sigma}(T)=\int_{1.8 \mathrm{eV}}^{2.4 \mathrm{eV}} d E_{\tau} \int_{0.6 \mathrm{eV}}^{1.4 \mathrm{eV}} d E_{t^{\prime}} S\left(E_{t^{\prime}}, T, E_{\tau}\right)
$$

In the upper panel of Fig. 6 , we show this curve for the case of zero coupling $K$. Again, we use the $K=0$ as a reference system where EEA does not take place. The displayed curve is obtained by subtracting the background signal, which stems from contributions which do not depend on the population time $T$. The signal oscillates with a period of approximately $T_{D}=2 \pi \hbar /\left(D_{3}-D_{1}\right)=20.4 \mathrm{fs}$. This is just what is expected from the energy separation $D_{3}-D_{1}=0.2 \mathrm{eV}$ obtained for $K=0$. In the present case, where the EEA process is blocked, the oscillations occur around a value of zero (i.e., the background signal).

If the coupling is switched on, the annihilation pathway is activated, and the curve changes substantially. This is documented in the lower panel of Fig. 6. The oscillations no longer take place around the background but are superimposed on a decaying signal. For the chosen value of $K=0.1 \mathrm{eV}$, the oscillation period is $11.9 \mathrm{fs}$. Its dependence on the coupling constant $K$ can be calculated from the energy 


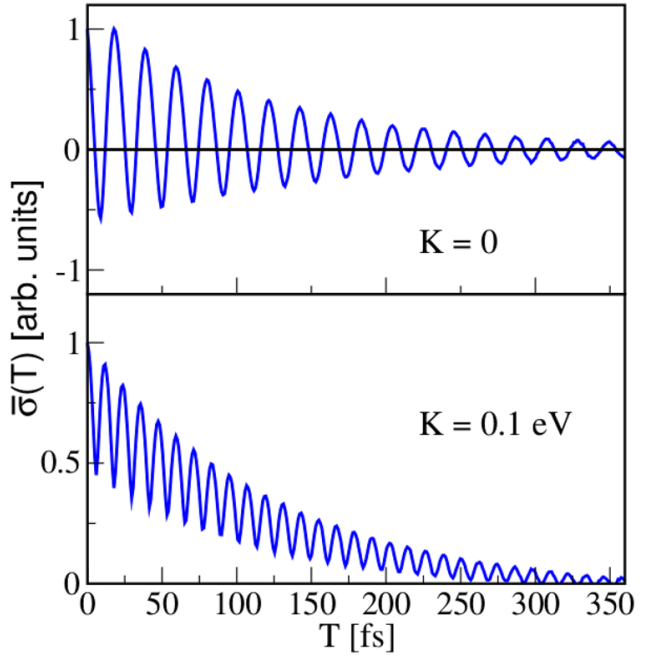

FIG. 6. Integrated signal $\bar{\sigma}(T)$ as a function of the population time $T$. The $T$ independent background signal obtained for long times is subtracted. Upper panel: If no coupling $K$ is active only the direct excitation pathway influences the decay of the signal. The signal oscillates roughly around a value of zero (indicated by the horizontal line). Lower panel: For non-zero coupling $K$, where EEA is present, the oscillations are superimposed on a decaying signal.

difference between $D_{3}-D_{1}$ using Eqs. (9) and (11). For example, if $E_{e e}=E_{g f}$, it scales with $\sqrt{8} \mathrm{~K}$.

A comparison of the two cases depicted in Fig. 6 suggests that, for a dimeric system, the fifth-order signal can indeed be used to characterize the presence of EEA.

\section{SUMMARY}

We studied a model dimer system where the focus was on the characterization of exciton-exciton annihilation via fifth-order twodimensional optical spectroscopy. In the model, relaxation of laserexcited states proceeds via two pathways. First, two-photon absorption leads to the population of a manifold of second excited states which directly relax. The second path includes exciton-exciton annihilation, where an additional population of the decaying states is prompted by exciton-exciton interaction. A complete disentanglement of the two pathways is not possible because the second step of EEA is identical to the one present in the direct excitation pathway.

We calculated 2D spectra in the spectral range where signatures of the EEA appear. In particular, energy-integrated spectra were determined as a function of the population time. The time interval spans the entire time scale where relaxation takes place. In a comparison of signals with and without EEA, we found that the decay curves exhibit pronounced differences. Without EEA an oscillating signal can be constructed where the oscillations are damped and occur on top of a background signal. Including EEA results in damped oscillations which are superimposed on a decaying signal. We thus conclude that, in principle, annihilation processes can be traced via 2D spectroscopy for the smallest molecular aggregate.
In going from the dimer to larger aggregates one faces a different situation. In the dimer, the interacting excitons are prepared on neighboring sites so that EEA starts immediately after laser preparation of the excitonic states. In a more extended system, excitons may be prepared on sites which are far apart. Then, the exciton-exciton interaction will only be effective if the excitons diffuse towards each other and finally meet. Thus, in larger systems the phenomena related to the existence of exciton-exciton interactions will appear time delayed. ${ }^{16}$ This delay is not present in the dimer case where laser excitation prepares excitons on the two monomers residing right next to each other. Investigations on larger aggregates are under way.

\section{ACKNOWLEDGMENTS}

This work was funded by the Deutsche Forschungsgemeinschaft (DFG, German Research Foundation)-Grant Nos. 220404594 (T.B.) and 207601309 (V.E.), and the European Research Council (ERC)—Grant No. 614623 (T.B.).

\section{REFERENCES}

${ }^{1}$ Y. Zaushitsyn, K. G. Jespersen, L. Valkunas, V. Sundström, and A. Yartsev, Phys. Rev. B 75, 195201 (2007).

${ }^{2}$ D. C. Dai and A. P. Monkman, Phys. Rev. B 87, 045308 (2013).

${ }^{3}$ M. A. Stevens, C. Silva, D. M. Russell, and R. H. Friend, Phys. Rev. B 63, 165213 (2001).

${ }^{4}$ S. Cook, H. Liyuan, A. Furube, and R. Katoh, J. Phys. Chem. C 114, 10962 (2010).

${ }^{\mathbf{5}}$ A. Lewis, A. Ruseckas, O. Gaudin, G. Webster, P. Burn, and I. Samuel, Org. Electron. 7, 452 (2006).

${ }^{6}$ D. Peckus, A. Devižis, D. Hertel, K. Meerholz, and V. Gulbinas, Chem. Phys. 404, $42(2012)$

${ }^{7}$ S. Gòlinas, J. Kirkpatrick, I. A. Howard, K. Johnson, M. W. B. Wilson, G. Pace, R. H. Friend, and C. Silva, J. Phys. Chem. B 117, 4649 (2013).

${ }^{8}$ R. S. Knox, Theory of Excitons, Solid State Physics, Advances in Research and Applications, Vol. 5 (Academic Press, New York, 1963).

${ }^{9}$ A. S. Davydov, Theory of Molecular Excitons (Plenum, New York, 1971).

${ }^{10} \mathrm{~V}$. May and O. Kühn, Charge and Energy Transfer Dynamics in Molecular Systems, 3rd ed. (Wiley VCH, Weinheim, 2011).

${ }^{11}$ H. Marciniak, X.-Q. Li, F. Würthner, and S. Lochbrunner, J. Phys. Chem. A 115, 648 (2011).

${ }^{12}$ S. Wolter, J. Aizezers, F. Fennel, M. Seidel, F. Würthner, O. Kühn, and S. Lochbrunner, New J. Phys. 14, 105027 (2012).

${ }^{13}$ F. Fennel and S. Lochbrunner, Phys. Rev. B 92, 140301 (2015).

${ }^{14}$ S. F. Völker, A. Schmiedel, M. Holzapfel, K. Renziehausen, V. Engel, and C. Lambert, J. Phys. Chem. C 118, 17467-17482 (2014).

${ }^{15}$ B. Brüggemann and T. Pullerits, New J. Phys. 13, 025024 (2011).

${ }^{16}$ J. Dostál, F. Fennel, F. Koch, S. Herbst, F. Würthner, and T. Brixner, Nat. Commun. 9, 2466 (2018).

${ }^{17}$ W. P. Aue, E. Bartholdi, and R. R. Ernst, J. Chem. Phys. 64, 2229 (1976).

${ }^{18}$ R. R. Ernst, G. Bodenhausen, and A. Wokaun, Principles of Nuclear Magnetic Resonance in One and Two Dimensions (Clarendon Press, Oxford, 1987).

${ }^{19}$ P. Hamm, M. Lim, and R. M. Hochstrasser, J. Phys. Chem. B 102, 6123 (1998).

${ }^{20}$ M. Khalil, N. Demirdöven, and A. Tokmakoff, J. Phys. Chem. A 107, 5258 (2003).

${ }^{21}$ J. Bredenbeck, J. Helbing, C. Kolano, and P. Hamm, Chem. Phys. Chem. 8, 1747 (2007).

${ }^{22}$ M. Cho, Two-Dimensional Optical Spectroscopy (CRC Press, Baton Rouge, 2009).

${ }^{23}$ I. J. Finkelstein, J. Zheng, H. Ishikawa, S. Kim, K. Kwak, and M. D. Fayer, Phys. Chem. Chem. Phys. 9, 1533 (2007). 
${ }^{24} \mathrm{P}$. Hamm and M. Zanni, Concepts and Methods of $2 \mathrm{D}$ Infrared Spectroscopy (Cambridge University Press, Cambridge, 2011).

${ }^{25}$ M. D. Fayer, Ultrafast Infrared Vibrational Spectroscopy (CRC, Boca Raton, 2013).

${ }^{26}$ J. D. Hybl, A. W. Albrecht, S. M. G. Faeder, and D. M. Jonas, Chem. Phys. Lett. 297, 307 (1998).

${ }^{27}$ D. M. Jonas, Annu. Rev. Phys. Chem. 54, 425 (2003).

${ }^{28}$ P. Tian, D. Keusters, Y. Suzaki, and W. S. Warren, Science 300, 1553 (2003).

${ }^{29}$ M. L. Cowan, J. P. Ogilvie, and R. J. D. Miller, Chem. Phys. Lett. 386, 184 (2004).

${ }^{30}$ T. Brixner, T. Mančal, I. Stiopkin, and G. R. Fleming, J. Chem. Phys. 121, 4221 (2004).

${ }^{31}$ T. Brixner, J. Stenger, H. M. Vaswani, M. Cho, R. E. Blankenship, and G. R. Fleming, Nature 434, 625 (2005).

${ }^{32}$ F. D. Fuller and J. P. Ogilvie, Annu. Rev. Phys. Chem. 66, 667 (2015).

${ }^{33}$ P. Nuernberger, S. Ruetzel, and T. Brixner, Angew. Chem., Int. Ed. Engl. 54, 11368 (2015).

${ }^{34}$ V. Szöcs, T. Palszegi, V. Lukes, J. Sperling, F. Milota, W. Jakubetz, and H. F. Kauffmann, J. Chem. Phys. 124, 124511 (2006).

${ }^{35}$ G. S. Engel, T. R. Calhoun, E. L. Read, T.-K. Ahn, T. Mančal, Y.-C. Cheng, R. E. Blankenship, and G. R. Fleming, Nature 446, 782 (2007).

${ }^{36}$ E. L. Read, G. S. Schlau-Cohen, G. S. Engel, J. Wen, R. E. Blankenship, and G. R. Fleming, Biophys. J. 95, 847 (2008).

${ }^{37}$ T. Mančal, N. Christensson, V. Lukes, F. Milota, O. Bixner, H. F. Kauffmann, and J. Hauer, J. Phys. Chem. Lett. 3, 1497 (2012).

${ }^{38}$ A. Halpin, P. J. M. Johnson, R. Tempelaar, R. S. Murphy, J. Knoester, T. Jansen, and R. J. D. Miller, Nat. Chem. 6, 196 (2014).

${ }^{39}$ M. Kullmann, S. Ruetzel, J. Buback, P. Nuernberger, and T. Brixner, J. Am. Chem. Soc. 133, 13074 (2011).

${ }^{40}$ O. Bixner, V. Lukes, T. Mančal, J. Hauer, F. Milota, M. Fischer, I. Pugliesi, M. Bradler, W. Schmid, E. Riedle et al., J. Chem. Phys. 136, 204503 (2012).

${ }^{41}$ K. W. Stone, K. Gundogdu, D. B. Turner, X. Li, S. T. Cundiff, and K. A. Nelson, Science 324, 1169 (2009).

${ }^{42}$ G. Nardin, T. M. Autry, K. L. Silverman, and S. T. Cundiff, Opt. Express 21, 28617 (2013).

${ }^{43}$ M. Kasha, Radiat. Res. 20, 55 (1963).

${ }^{44}$ M. Kasha, H. R. Rawls, and M. A. El-Bayoumi, Pure Appl. Chem. 11, 371 (1965).

${ }^{45}$ T. Renger and V. May, Phys. Rev. Lett. 78, 3406 (1997).

${ }^{46}$ B. Brüggemann, J. L. Herek, V. Sundström, T. Pullerits, and V. May, J. Phys. Chem. B 105, 11391 (2001).

${ }^{47}$ V. May, J. Chem. Phys. 140, 054103 (2014).
${ }^{48}$ K. Hader, V. May, C. Lambert, and V. Engel, Phys. Chem. Chem. Phys. 18, 13368 (2016).

${ }^{49}$ K. Hader, C. Consani, T. Brixner, and V. Engel, Phys. Chem. Chem. Phys. 19, 31989 (2017).

${ }^{50}$ E. S. Medvedev and V. I. Osherov, Radiationless Transitions in Polyatomic Molecules (Springer, Berlin, 1995).

${ }^{51} \mathrm{M}$. Keß, G. Worth, and V. Engel, J. Chem. Phys. 145, 084305 (2016).

${ }^{52}$ J. Wehner and V. Engel, Phys. Chem. Chem. Phys. 18, 32910 (2016).

${ }^{53}$ M. Schröter, S. Ivanov, J. Schulze, S. Polyutov, Y. Yani, T. Pullerits, and O. Kühn, Phys. Rep. 567, 1 (2015).

${ }^{54}$ R. L. Fulton and M. Gouterman, J. Chem. Phys. 41, 2280 (1964).

${ }^{55}$ B. Engels and V. Engel, Phys. Chem. Chem. Phys. 19, 12604 (2017).

${ }^{56} \mathrm{R}$. Tempelaar, T. L. C. Jansen, and J. Knoester, J. Phys. Chem. Lett. 8, 6113 (2017).

${ }^{57}$ L. Bruder, A. Eisfeld, U. Bangert, M. Binz, M. Jakob, D. Uhl, M. Schulz-Weiling, E. R. Grant, and F. Stienkemeier, Phys. Chem. Chem. Phys. 21, 2276 (2019).

${ }^{58}$ K. Mølmer, Y. Castin, and J. Dalibard, J. Opt. Soc. Am. B 10, 524 (1993).

${ }^{59}$ D. E. Makarov and H. Metiu, J. Chem. Phys. 111, 10126 (1999).

${ }^{60}$ J. Albert, M. Falge, M. Keß, J. Wehner, P. Zhang, A. Eisfeld, and V. Engel, J. Chem. Phys. 142, 212440 (2015).

${ }^{61}$ M. Kasha, Faraday Discuss. Chem. Soc. 9, 14 (1950).

${ }^{62} \mathrm{~K}$. Blum, Density Matrix Theory and Applications, 3rd ed. (Springer, Heidelberg, 2012).

${ }^{63}$ M. Keß and V. Engel, Chem. Phys. Lett. 650, 41 (2016).

${ }^{64}$ S. Mukamel, Principles of Nonlinear Optical Spectroscopy (Oxford University Press, New York, 1995).

${ }^{65}$ W. Domcke and G. Stock, Adv. Chem. Phys. 100, 1 (1997).

${ }^{66}$ S. Mukamel, Annu. Rev. Phys. Chem. 51, 691 (2000).

${ }^{67}$ D. J. Tannor, Introduction to Quantum Mechanics: A Time-Dependent Perspective (University Science Books, Sausalito, 2007).

${ }^{68}$ P. Kjellberg, B. Brüggemann, and T. Pullerits, Phys. Rev. B 74, 024303 (2006).

${ }^{69}$ A. Nemeth, F. Milota, T. Mančal, V. Lukeš, J. Hauer, H. F. Kauffmann, and J. Sperling, Chem. Phys. Lett. 459, 94 (2008).

${ }^{70}$ T. Mančal, A. Nemeth, F. Milota, V. Lukeš, J. Hauer, H. F. Kauffmann, and J. Sperling, J. Chem. Phys. 132, 184514 (2010).

${ }^{71}$ D. B. Turner, K. E. Wilk, P. M. G. Curmi, and G. D. Scholes, J. Phys. Chem. Lett. 2, 1904 (2011).

${ }^{72}$ K. Franstedt and G. S. Engel, Chem. Phys. 403, 59 (2012).

${ }^{73}$ V. Tiwari, W. K. Peters, and D. M. Jonas, Proc. Natl. Acad. Sci. U. S. A. 110, 1203 (2013). 